\title{
Detection of Indonesian Vehicle Plate Location using Harris Corner Feature Detector Method
}

\author{
Fawwaz Ali Akbar ${ }^{1}$, Hendra Maulana \\ Department of Informatics \\ Universitas Pembangunan Nasional "Veteran" Jawa Timur \\ Surabaya, Indonesia \\ ${ }^{1}$ fawwaz.ali@gmail.com
}

\author{
Anny Yuniarti ${ }^{2}$ \\ Department of Informatics \\ Institut Teknologi Sepuluh Nopember \\ Surabaya, Indonesia \\ 2anny@if.its.ac.id
}

\begin{abstract}
Intelligent transportation systems are now starting to develop. One aspect of the intelligent transportation support system is how to recognize a vehicle. Vehicle plate recognition is an important function of this system. Plate detection systems have two problems, namely where the plate is located and how large the size is. Harris, Eigen and FAST methods have a high production value in detecting the number plate location. This is because the theoretical background is relatively focused on the angle. The Harris Corner detection system is often used because it can produce consistent values in rotating image and which has a lot of noise in an image. Morphological filters openings and closings can be seen as transformations with a structuring element which locally adapts its shape to the image structures, and therefore have lovely filtering capabilities. In this paper, we present the Indonesian plate localization based on text segmentation of unstructured standard plates with morphology filters openings and closings on pre-processing and feature detector Harris Corner method. Harris Corner method is used to detect corner points of the text, as well as using the Euclidean Distance algorithm to match features. The proposed result method produces an accuracy of $98.98 \%$, a precision of $65.57 \%$ and a recall value of $73.55 \%$. For further research, it needs to be compared with other methods to get the best results.
\end{abstract}

Keywords-Harris Corner; Morphological Filters; Plate Localization; Euclidean Distance

\section{INTRODUCTION}

In recent years, with the increasing number of complex forms of transportation and modern transportation networks, intelligent transportation systems play an essential role in our daily lives. The function of the system is to monitor traffic, to record vehicle information and report ordinary traffic events. In general, plate detection systems have two problems, namely where the plate is located and how large size plate [1]. Usually, the position of the candidate character in the plate is first identified, and the box area of the plate is determined later. Based on observations [2] that certain characters in different number plates can be considered as duplicates of each other, arc models based on local features for plate detection are proposed because visual words generated from unsupervised groupings are sensitive to noise features from image background.

Harris Corner detection system is often used because it can produce consistent values in images that experience rotation, variations in lighting and that has a lot of noise in the image [3]. The extraction feature of Harris Corner is run by detecting the characteristics of an object in the form of an angle. Harris Corner algorithm works very well on positive and negative skewed images. Algorithms provide reliable results with various computational complexity [4].

Horak research declares Harris, Eigen and FAST methods have a high productivity value in detecting the number plate location [5]. This is because the theoretical background is relatively focused on the angle.

Other research declares the gray-scale opening and closing areas can be used as a single structure of the image, and therefore have excellent filtering capabilities [6]. The effect is compared to standard morphological filters. Some applications in image segmentation and hierarchical decomposition are also brief.

Grey-scale area openings and closings can be seen as transformations with a structuring element which locally adapts its shape to the image structures, and therefore have very nice filtering capabilities. Their effect is compared to that of more standard morphological filters. Some applications in image segmentation and hierarchical decomposition are also briefly described.

In this paper, we present the Indonesian plate localization based on text segmentation of unstructured standard plates with morphology filtering openings and closings on pre-processing and feature detector Harris Corner method. The performance of the proposed system is tested on our own set of image data.

\section{RELATED WORK}

This research using morphology filtering at pre-processing and corner point description to detect license plate location. In this section, an overview of the proposed method that implements feature extraction is presented. The proposed system combines the following sub-systems: Feature Extraction Templates, Preprocessing, Harris Corner Detection, Feature Matching, and Mark Selected Corner Point. Proposed system flow is presented in Fig. 1. 


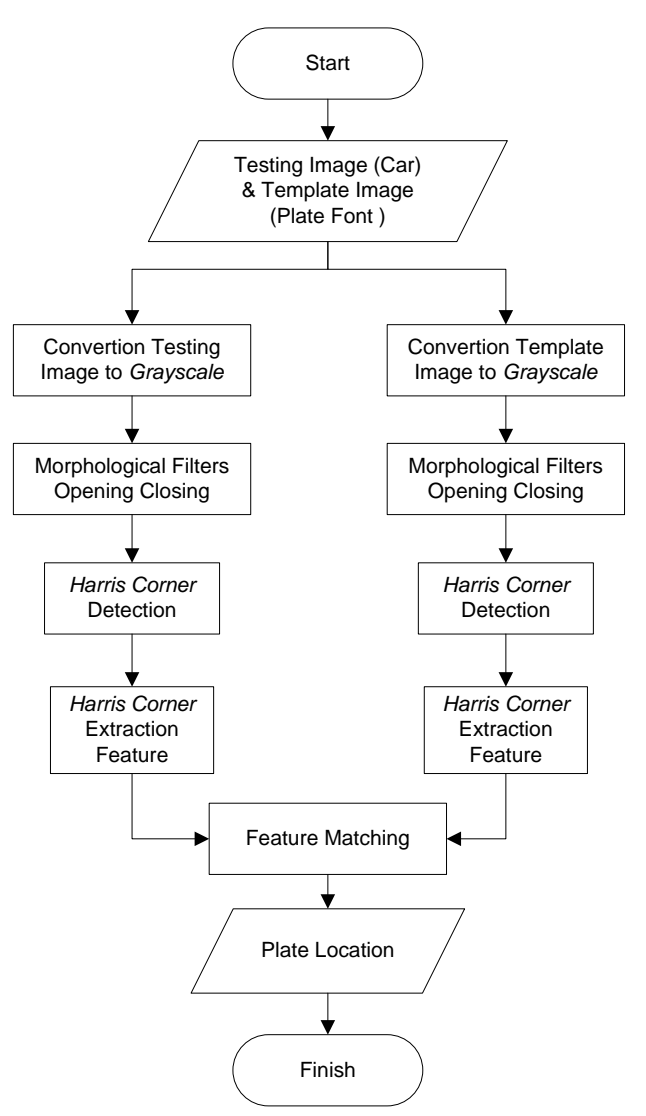

Fig. 1 Proposed System Flow

\section{A. Template Extraction}

Template extraction, using an image of Indonesian standard font of vehicle license plate, keypoint detection is performed. To ensure all templates can be used for feature matching, keypoint search and feature extraction are performed. Keypoint search and feature extraction methods used in this Harris Corner process. Template image with key point, then used in the process of matching features with a testing image.

\section{B. Pre-Processing}

Testing image datasets as 20 images are still in the form of original images taken by the author. The dataset still has a lot of noise that must be improved. The process of processing data at this preprocessing stage, each testing image is conferred to grayscale, then morphology filtering is done to eliminate noise using opening and closing operations.

\section{Deteksi Harris Corner}

Detection feature of the Harris Corner method is used to detect corner points. The Harris Corner algorithm is used to extract features from testing images that will be matched with the corner point template feature. The Harris Corner feature detection is shown in Fig. 2.

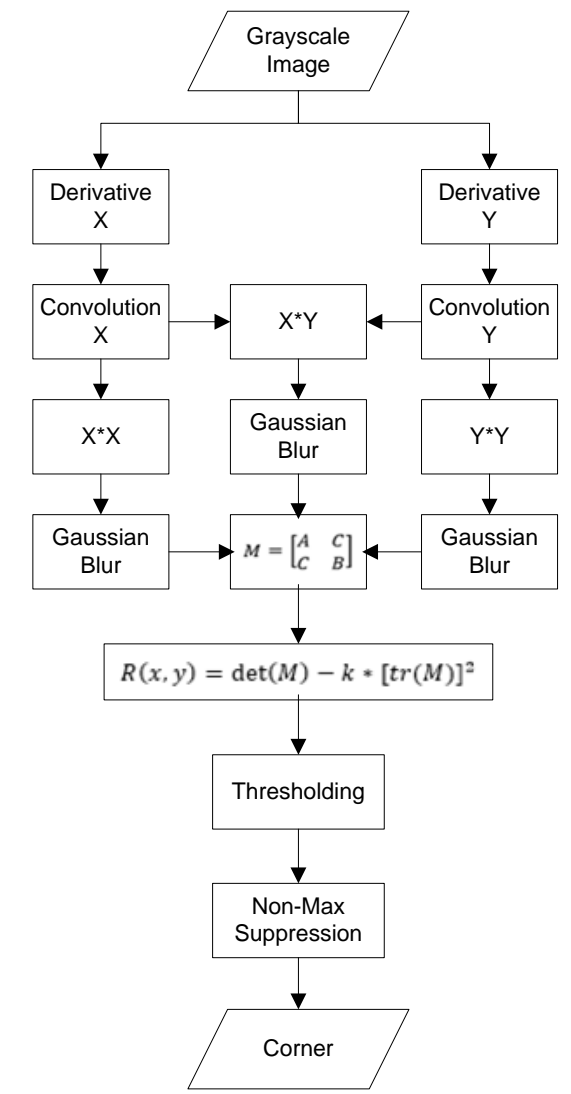

Fig. 2 Harris Corner Flow

In Corner Detection, three derivative images are needed, namely Derivative $\mathrm{X}$, Derivative $\mathrm{Y}$, Derivative $\mathrm{X}^{*} \mathrm{Y}$. Three convolution images using the Prewitt operator in the previous process will be blurred with the Gaussian operator so that a slightly blurry image will appear.

For each point on the image $2 \times 2 \mathrm{M}$ matrix is built and calculates the Plessey operator. Where $\mathrm{A}$ is the derivative image $\mathrm{X}$ that has been blurred, $\mathrm{B}$ derivative image $\mathrm{Y}$ that has been blurred, and $\mathrm{C}$ is the $\mathrm{X}^{*} \mathrm{Y}$ derivative image that has been blurred from the previous Gaussian Blur process. After that, the Plessey value of each pixel can be obtained. det $(\mathrm{M})$ is the determinant of the matrix $\mathrm{M}$ that is $(\mathrm{A} * \mathrm{~B})-(\mathrm{C} * \mathrm{C})$ and $\operatorname{tr}(\mathrm{M})$ is the Trace of the matrix $M$ that is $(A+B)$.

Thresholding is used to partition an image by setting the intensity value of all pixels that are greater than the threshold value of $\mathrm{T}$ as an object and which is smaller than the threshold value of $\mathrm{T}$ as the background. The Non Maximum Suppression process which is similar to the process of thinning (downsizing) is done to determine the edge pixels with the position closest to the location of the change in pixel value between the number of edge pixels detected. If the Plessey value of a given point is the local maximum value in a $3 \times 3$ region, it can be concluded that this point is a corner point

\section{Feature Matching}

At the matching stage, after keypoint location, keypoint descriptor and image for training and testing images are obtained, then the correlation value is calculated using the Euclidean Distance algorithm. An image can be presented as a 
matrix, so that it can be said that an image is considered the same if each cell of the matrix has the same element value in the same row and column position.

In this research, testing images will look for similarities with a number of template images to find the location of the vehicle number plate. The algorithm used is to apply the Euclidean Distance formula equation.

\section{E. Mark Selected CornerPoint}

After character features in the testing image are identified, the geometric context is suitable to find the location of the plate. A boundary box will be estimated to include a plate by determining the boundary lines up, down, left, and right in sequence. After that, a rough estimate of the left and right boundary lines is obtained. On the plate, the ratio of plate width to height is constant. When the height of the plate is estimated, it can also be obtained the width of the plate.

\section{F. Dataset}

Dataset used in this study is image testing of a car taken by the author himself, as many as 20 images, each of which has a resolution of $4208 \times 3120$ pixels and an RGB color band. And image Indonesian police standard vehicle number font template.

\section{G. Testing Scenario \& Analysis}

The scenario in this study consists of several stages, following the stages of trial and analysis scenarios.

- Test the proposed method (Pre-processing with morphology filtering opening closing and Harris Corner Detection)

- Perform feature matching corner points (keypoint location, keypoint descriptor, and image) for training and testing images.

- Calculate accuracy, precision, recall and computation time from the results of the testing of the proposed method (Preprocessing with Morphology Filtering and Harris Corner Detection).

- Analysis of the results of experiments performed

\section{RESULT AND ANALYSIS}

This chapter explains the results of each extraction and its analysis. In the analysis of results will be written all the results of the trial implementation process and its analysis. Some of the results that will be presented include the results of making templates, the results of pre-processing, results of detection of Harris Corner, results of Mark and Mask Selected Corner Point, and Analysis.

\section{A. Template}

Before searching for corner point features, template image was changed to grayscale image first. Results of the corner point template feature are presented in Fig. 3.

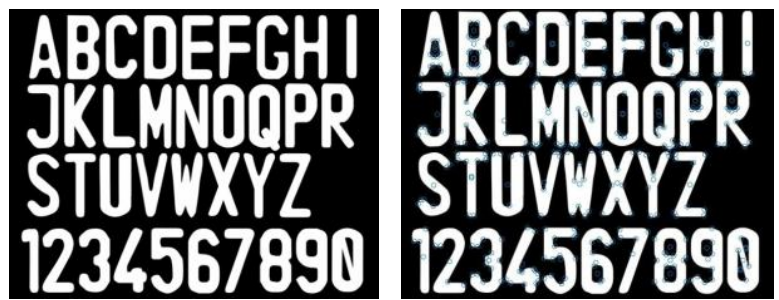

Template

Corner Point Template

Fig. 3 Template

\section{B. Pre-Processing Result}

On the testing image, preprocessing process will be carried out. Testing images will be configured to grayscale, then morphology filtering is done to eliminate noise using opening and closing operations. The results of testing image preprocessing are presented in Fig. 4.
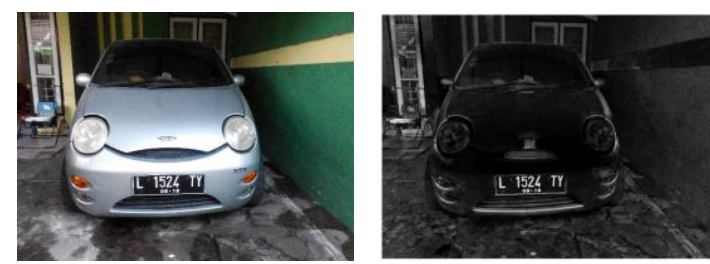

Fig. 4 Pre-Processing Result

\section{Harris Corner Detection Result}

Harris Corner algorithm is used to extract features from testing images that will be matched with the corner point template feature. The detection results of the Harris Corner feature are presented in Figure 5.

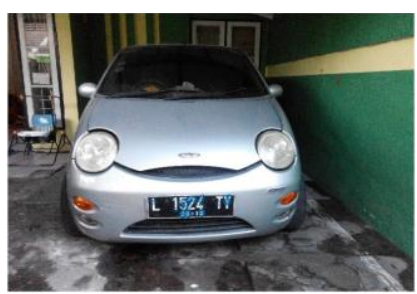

Fig. 5 Harris Corner Detection Result

\section{Feature Matching}

Matching based keypoint location and keypoint descriptor on testing images and template image. An image can be presented as a matrix so that it can be said that an image is considered the same if each cell of the matrix has the same element value in the same row and column position. Feature matching result is presented in Fig. 6.

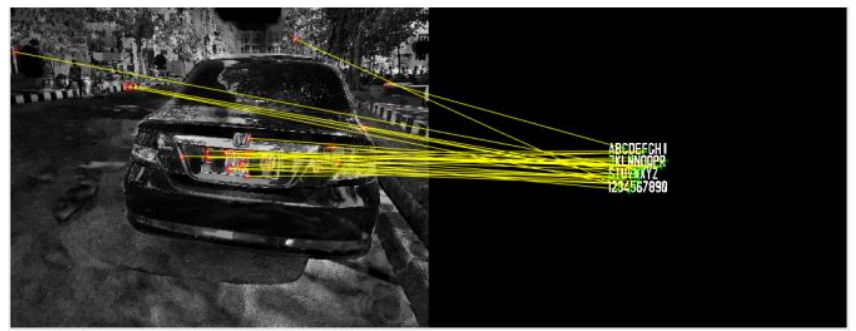

Fig. 6 Feature Matching Result 


\section{E. Mark Selected Corner Point}

After getting the matching feature pair, the testing image is marked. Marking is done based on the corner point feature. Test results of 20 testing data are presented in Fig. 7.

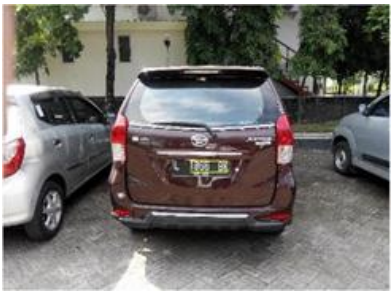

1

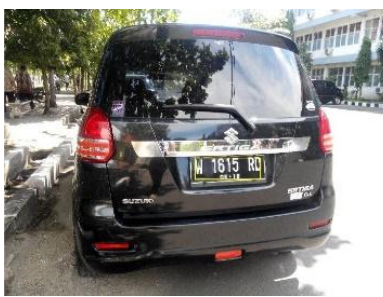

3

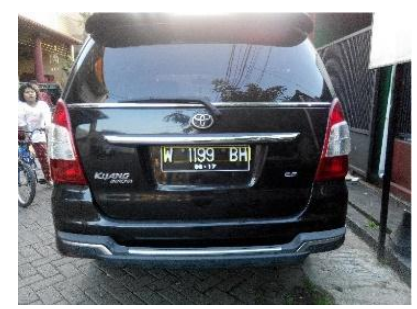

5

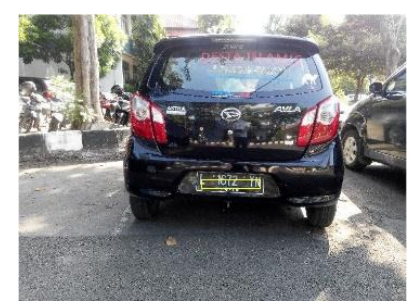

7

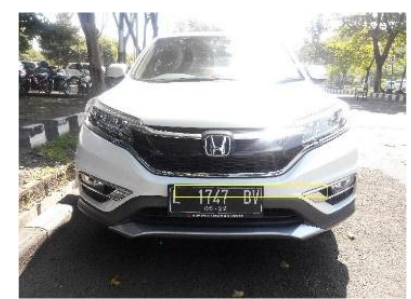

9

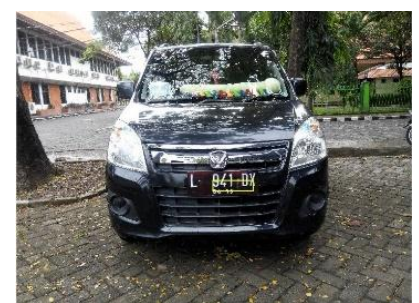

11

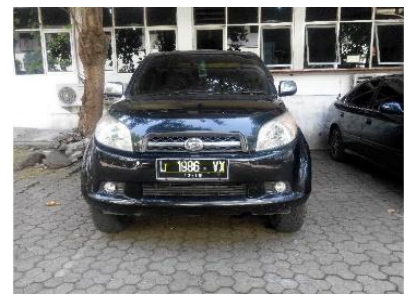

2

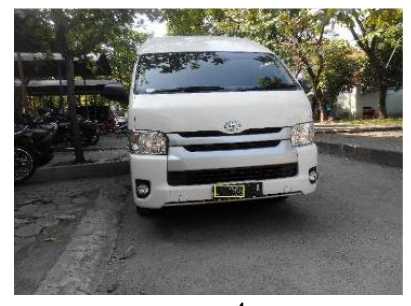

4

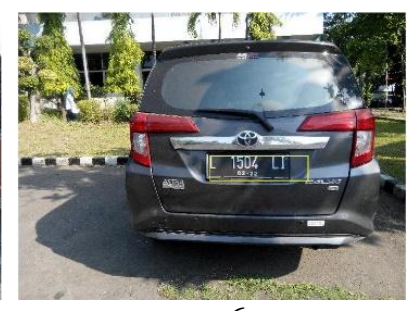

6

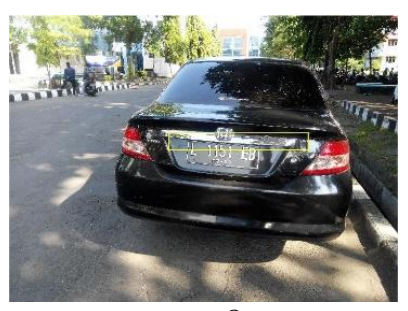

8

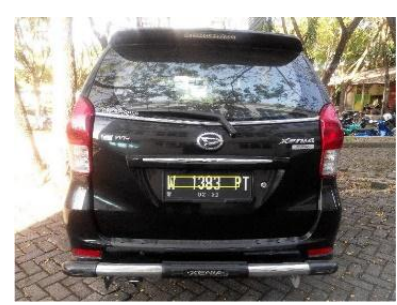

10

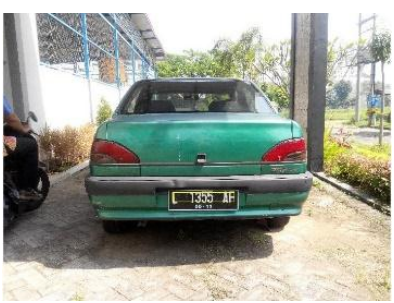

12

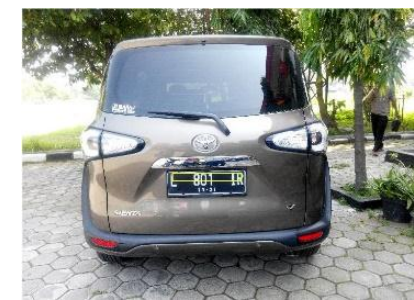

13

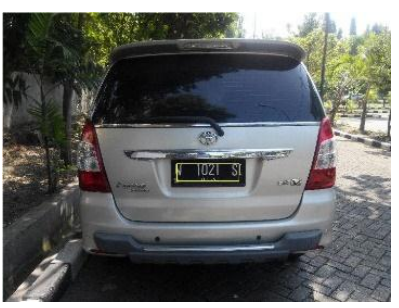

15

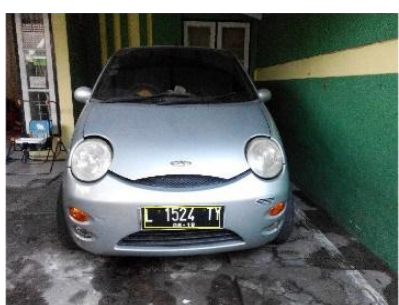

17

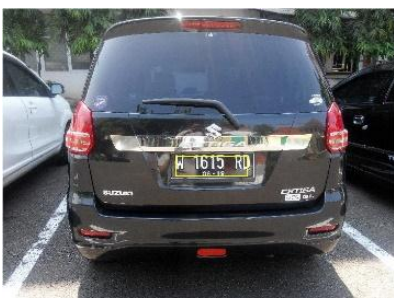

19

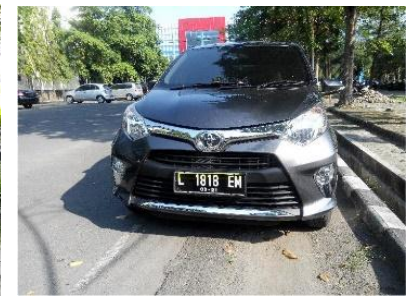

14

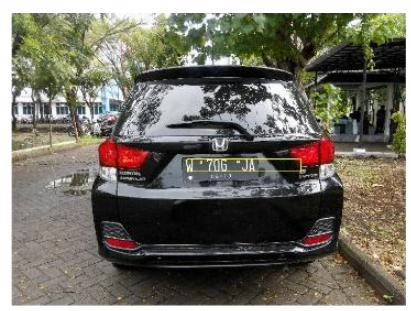

16

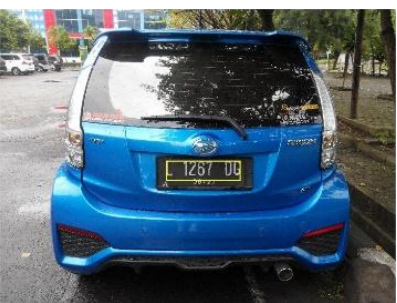

18

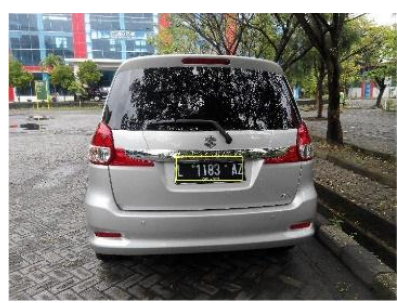

20
Fig. 7 Localization Plate Result

F. Analysis

After image plate testing location is obtained, accuracy, precision and recall will be calculated. The formula for calculating accuracy, precision and recall presented in (1).

$$
\begin{aligned}
& \text { accuracy }=\frac{T P+T N}{T P+T N+F P+F N} \\
& \text { precision }=\frac{T P}{T P+F P} \\
& \text { recall }=\frac{T P}{T P+F N}
\end{aligned}
$$

Calculation results of accuracy, precision and recall image testing presented in Table I. 
TABLE I. Result OF ACCURACy, PRECISION AND RECALL

\begin{tabular}{|c|c|c|c|}
\hline Image & Accuracy & Precision & Recall \\
\hline 1 & 98.6 & 46.17 & 100 \\
\hline 2 & 99.76 & 62.56 & 67.95 \\
\hline 3 & 99.58 & 92.16 & 72.16 \\
\hline 4 & 98.72 & 50.63 & 86.67 \\
\hline 5 & 98.83 & 87.28 & 95.84 \\
\hline 6 & 98.67 & 91.7 & 42.33 \\
\hline 7 & 99.51 & 47.42 & 68.3 \\
\hline 8 & 97.62 & 42.23 & 20.9 \\
\hline 9 & 98.41 & 53.53 & 33.71 \\
\hline 10 & 98.54 & 58.27 & 100 \\
\hline 11 & 99.39 & 33.31 & 69.72 \\
\hline 12 & 98.93 & 87.84 & 100 \\
\hline 13 & 99.69 & 60.77 & 100 \\
\hline 14 & 99.55 & 37.62 & 99.69 \\
\hline 15 & 99.65 & 94.65 & 68.25 \\
\hline 16 & 98.03 & 59.08 & 60.48 \\
\hline 17 & 98.18 & 37.8 & 100 \\
\hline 18 & 99.69 & 100 & 74.38 \\
\hline 19 & 99.45 & 89.29 & 62.38 \\
\hline 20 & 98.81 & 79.19 & 47.61 \\
\hline
\end{tabular}

\section{CONCLUSION}

In this research, Harris Corner method is used to detect corner points of text, as well as using the Euclidean Distance algorithm to match features. Detection Indonesian vehicle plate location with the filtering opening-closing morphology in preprocessing and Feature Detection Harris Corner method produces an accuracy of $98.98 \%$, a precision of $65.57 \%$ and a recall value of $73.55 \%$. For further research, it needs to be compared with other methods to get the best results.

\section{REFERENCES}

[1] G. S. Hsu, J. C. Chen and Y. Z. Chung, "Application-oriented license plate recognition," IEEE transactions on vehicular technology, 62(2), pp.552-561, 2013.

[2] Z. Wengang, et al, "Principal visual word discovery for automatic license plate detection," IEEE transactions on image processing 21.9 4269-4279, 2012.

[3] C. Harris and M. Stephen, "A Combined Corner and Edge Detector," Procedings of the Alvey Vision Conference, pp.147-151, 1988

[4] N.D. Modi, et al, "Skew correction for vehicle license plates using principal component of Harris Corner Feature," Communication Systems and Network Technologies (CSNT), 2011 International Conference on. IEEE, 2011.

[5] Horak, Karel, J. Klecka, and P. Novacek, "License plate detection using point of interest detectors and descriptors," Telecommunications and Signal Processing (TSP), 2016 39th International Conference on. IEEE, 2016.

[6] L. Vincent, "Morphological area openings and closings for grey-scale images," Shape in Picture. Springer, Berlin, Heidelberg, 197-208, 1994. 